Research Article

\section{Assessment of shoulder pain and somatic dysfunction in young competitive swimmers: Preventive Osteopathic Manipulative Treatment}

\author{
Fanny Granger ${ }^{1}$, Marion Etevenard ${ }^{2}$, Jean-Pascal Kily ${ }^{1}$ and Martin \\ Garet ${ }^{1,3 *}$ \\ ${ }^{1}$ Centre International d'ostéopathie, Saint-Etienne, France \\ ${ }^{2}$ Osteopath Private Practice, Personal trainer, Genève, Suisse, Switzerland \\ ${ }^{3}$ Service de Physiologie Clinique et de l'Exercice, Laboratoire SNA EPIS, Saint-Etienne, France
}

\section{Abstract}

Context: Shoulder pain is one of the most frequent reported complaints in intensive competitive swimming. The so-called 'swimmers' shoulder' has been widely explored and has been reported sometimes without specific reference to contributing mechanisms or structures. Somatic dysfunction is defined as an impaired or altered function of related components of the somatic system and may appear in the early stage of pain feeling.

Aim: To evaluate somatic dysfunctions in a group of young competitive swimmers with and without shoulder pain and its relationship with the shoulder's mobility along with the efficacy of an osteopathic manipulative treatment (OMT) on shoulder's mobility, pain, and comfort of swimming.

Material and method: 20 competitive swimmers (14.6 \pm 1.3 ys; $11.6 \pm 2.4$ hs.wk-1) were divided into two groups, with and without shoulder pain (SPG/CG). Before and after light touch/ $\mathrm{OMT}$, and 1 week later, somatic dysfunctions, shoulder's range of mobility, pain, and swimming comfort were assessed by 2 independent osteopaths.

Results: Somatic dysfunctions were observed in both groups without significant differences in the number or localization and were independent of severity of pain. In the SPG, pain decreased significantly after OMT $(6.1 \pm 1.9$ vs. $3.9 \pm 1.8 ; p=0.001)$ and remained stable 1-week later $(P$ = NS). Shoulder's mobility was lower on the aching shoulder in the "shoulder pain" group when compared to the control group on flexion and abduction tests but not on extension or adduction tests. Following OMT, only abduction improved when compared to light touch. Comfort in swimming was reported as "better" in both OMT/light touch groups.

Conclusion: There is no difference between light touch and OMT as both decreased pain and increased comfort in swimming but abduction range of motion only improved in the OMT group.

\section{More Information}

*Address for Correspondence: Martin Garet Service de Physiologie Clinique et de l'Exercice, Laboratoire SNA EPIS, Saint-Etienne, France, Tel: +33674193735; +33477828300;

Email: martingaret@hotmail.com

Submitted: 05 July 2019

Approved: 08 August 2019

Published: 09 August 2019

How to cite this article: Granger F, Etevenard M, Kily JP, Garet M. Assessment of shoulder pain and somatic dysfunction in young competitive swimmers: Preventive Osteopathic Manipulative Treatment. J Sports Med Ther. 2019; 4: 061-066. DOI: dx.doi.org/10.29328/journal.jsmt.1001045

Copyright: @ 2019 Granger F, et al. This is an open access article distributed under the Creative Commons Attribution License, which permits unrestricted use, distribution, and reproduction in any medium, provided the original work is properly cited

Keywords: Swimming; Shoulder; Prevention; Mobility

Check for updates

\section{Introduction}

Swimming is a very popular sport practiced by million individuals worldwide. Among many physical activities, it is a great form of all-round exercise. When reasonably practiced, it appears to be a good activity in order to be more active and to stay healthy, whatever the age or ability [1]. It is a cyclic, highly repetitive sport combining endurance, velocity, strength, power, and control in a non-weight-bearing environment with important resistive forces. Propulsion is mainly driven by the upper body with repetitive overhead movements.
Up to $90 \%$ of the forward propulsive power in swimming comes from the upper extremities [2]. Combined to increasing training loads and/or inappropriate technique, along with an early involvement in this sport, this contributes to the appearance of shoulder pain and injuries, also known as the "swimmers' shoulder" describing a general syndrome with anterior shoulder pain elicited by repetitive impingement of the rotator cuff under the coracoacromial arch. In fact, shoulder pain appears to be the most common musculoskeletal complaint among swimmers. More precisely, it has been reported to be as high as $91 \%$ in competitive swimmers with 
$23 \%$ to $38 \%$ experiencing shoulder injuries each year [3-6]. As competitive swimming usually begins between 8 to 10 years old, even in 10-18 years old swimmers, shoulder pain has also been reported up to $43 \%$ [7]. In this same study, $66 \%$ of senior development swimmers, and $73 \%$ of elite swimmers reported a history of pain in the shoulders [7].

Training load and the cumulated years of swimming, along with the use of specific material (swimming paddles for example), inadequate periodization or inadequate technique are among many factors explaining the cause of shoulder pain and injury. Injury often occurs in swimmers' shoulders because long-term swimming puts excessive overhead arm movement on the shoulder, which has been shown to predispose the shoulder to mechanical impingement, glenohumeral instability, thoracic outlet syndrome, microtrauma, laxity, and fatigue [4,8-11]. A recent review by Heinlein explored the biomechanics of each stroke and their respective variations in the presence of pain [12]. This review referred to Sein's studies reporting that supraspinatus tendinopathy seemed to be the most common cause of pain in the swimmer's shoulder $[13,14]$. However, the review also pointed out the heterogeneity of the causes and that the complexity of the intervention [12]. This was also supported in another review by Struyf regarding the musculoskeletal dysfunctions associated with the "swimmers' shoulder" [15]. The review also pointed out the heterogeneity of the "swimmers' shoulder" and the lack of knowledge regarding the etiology. Therefore, defining successful interventions remains difficult as there is still a lack of knowledge regarding the best methods of prevention and treatment despite the known prevalence of shoulder injuries in swimmers [4]. Treatment recommendations are multiple and include exercise therapy like resistive exercise, neuromuscular retraining, stretching, or taping [16]. Treatment might also be surgery is specific cases but its efficacy versus physical therapy can still be discussed [17]. Both treatment and prevention strategies still remain to be explored.

Within manual therapies and prevention strategies, osteopathic manipulative treatment (OMT) has been proposed to prevent injuries or optimize performance [18-20]. In 124 collegiate cross-country athletes followed during several seasons, OMT yielded statistically significant decrease in the cumulative annual incidence of stress fractures in men [18]. Associating OMT and exercise therapy has also yielded positive results in the rehabilitation program of a high-level volleyball player, focusing on the importance of patient-centered practice and a multi-disciplinary approach [19]. Also, precompetition manipulative treatment has shown to be positively associated with improved performance among football players [20]. Methodological limitations remained in the later study though [20].

Somatic dysfunction is defined as an impaired or altered function of related components of the somatic system including skeletal, arthrodial, and myofascial structures and related vascular, neural, and lymphatic elements [21]. Dysfunctions that can be palpated include changes in tissue texture, increased sensitivity to touch (hyperalgesia), altered ease or range of movement, and anatomic asymmetry or positional change, according to the TART criteria [22], standing for Tenderness-Asymmetry-Restricted motion-Tissue texture changes. At least two of these criteria of the previous four presented are needed to consider a somatic dysfunction. To our knowledge, no previous studies have explored the possible association between somatic dysfunctions and pain evolution with swimming comfort in young swimmers along with the preventive capabilities of osteopathic manipulative treatment (OMT).

Specifically, we set out to evaluate somatic dysfunctions in a group of young competitive swimmers with and without shoulder pain and their relationship with the shoulder's mobility along with the efficacy of an osteopathic manipulative treatment (OMT) on shoulder's mobility, pain, and comfort of swimming. To our knowledge, there are no studies to date that examine specifically the relationship between somatic dysfunction and shoulder pain incidence along with the effects of OMT.

\section{Population}

Twenty regional to national level age-group swimmers $\left(14.6 \pm 1.3\right.$ ys; $11.6 \pm 2.4$ hs.wk $\left.^{-1}\right)$ participated in the study and were divided into two groups, one with shoulder pain (SPG), one without any complaint (CG). The SPG was further divided into 2 groups for OMT (SPG-OMT) or light touch (SPG-LT). All participants were issued from local swimming teams and were given information about the study and given the option to participate voluntarily. All individuals interested in study participation signed along with their parents an informed consent form approved by the institutional review board. All were swimming at least 4 days per week. None had a history of traumatic event at the time of inclusion nor was taking any releaving pharmacological treatment nor had any counterindication to OMT. Participants were excluded from the study if they began the study and subsequently failed to meet all inclusion criteria.

\section{Operators}

Two final-year osteopathic student-examiners were trained and guided by supervising osteopathic physician-examiners. They were trained and experienced in the management of somatic dysfunction. The students completed their formal training (academic curriculum) and were evaluated by means of both a written evaluation and a practical evaluation given by the attending physicians. The study protocol provided a detailed written description of physical examination, assessment, diagnosis, and corresponding black-box treatment, depending on the somatic dysfunctions found. The intervention included osteopathic structural examination and OMT that focused on somatic dysfunction identified as described 
in Appendix A. The operators focused mainly on the thoracic spine, scapular belt and the upper extremities. Skeletal osteoarticular, myofascial and muscle somatic dysfunctions were mainly sought. Examiners were not allowed to deviate from the study protocol and were not allowed to communicate to each-other during the protocol.

\section{Design}

All participants were scheduled on a specific day, at the beginning of a usual training session and one week later. The 2 student-osteopaths did not see the same participants at week 1. Osteopathic examination as well as pain and shoulder mobility were assessed randomly by the two operators. The participants included in the SPG-OMT or SPG-LT groups were not informed of their repartition. OMT was performed in a "black-box design" focusing on the somatic dysfunctions identified. To maintain a nontraumatic focus and minimize the risk of harm, the OMT techniques consisted primarily of muscle energy and articulatory techniques, and occasionaly high-velocity, low-amplitude techniques. The number of somatic dysfunctions and their location were recorded.

In SPG-OMT or SPG-LT groups, swimming comfort was assed qualitatively at the end of the study. with 3 specific verbatims ("feeling better", "no change", "worse").

\section{Material and Methods}

Somatic dysfunctions were independently evaluated by one or the other examiner on both shoulders. The examiner assessed 24 structural landmarks and functions of the shoulder while the participant was standing. During the assessments, the examiner and the scribe were blinded to the previous medical record of each participant, to the participants' health history, and to previous structural findings. As dictated by the examiner, the findings from the osteopathic structural examination were recorded by the scribe on the data collection form. All positive findings and diagnosed somatic dysfunctions were then treated on site by the student-examiner or the attending physician-examiner.

Shoulder pain was assessed with a pain severity index

Appendix A: Main body areas and structures investigated for somatic dysfunctions by the examiners.

\begin{tabular}{|c|c|c|}
\hline Skeletal (osteo-articular) & Myofascial & Muscle \\
\hline Sterno-costo-clavicular & clavipectoral fascia & Teres minor \\
\hline Acromio-clavicular & $\begin{array}{c}\text { Pretracheal fascia } \\
\text { Gervical fascia / superficial } \\
\text { layer of deep cervical } \\
\text { fascia }\end{array}$ & Supra / infra spinatus \\
\hline Radial head & Thoracic spine (T1 - T12) & Rhomboideus major \\
\hline Ulna & & Rhomboideus minor \\
\hline Radius & & Pectoralis major \\
\hline Carpus bones & & Pectoralis minor \\
\hline & & Latissimus dorsi \\
\hline & & Trapezius \\
\hline & & Deltoideus \\
\hline & & Biceps \\
\hline
\end{tabular}

analogic scale ranging from 0 (no pain) to 10 (worst pain possible). The participants were asked to rate their pain that corresponds to the intensity experienced.

Swimming comfort was assed qualitatively with 3 specific verbatims regarding their feeling ("better", "unchanged", "worse"). The participants were asked to rate the comfort experienced one week following OMT or light touch.

In order to assess shoulder's range of mobility, functional movement were performed in flexion, extension, abduction and adduction. A goniometer was used to evaluate amplitude in degrees. Measurements were performed at the beginning and after one week.

\section{Statistical analysis}

Results are given as mean \pm SD for continuous variables. Normality of the distributions was assessed with a Kolmogorov-Smirnov test. Differences in pain or amplitude between and within groups were assessed using an unpaired/a paired Student t-test. The relationship between intensity of pain and the number of dysfunctions was assessed with Spearman's correlation test and linear regression. Comfort of swimming was assessed qualitatively and quantified in terms of number of verbatims expressed by the participants. Statistical significance was set for $p<0.05$.

\section{Results}

\section{Characteristics of the population}

Twenty swimmers completed the study, 13 with shoulder pain and 7 without pain. Seven swimmers were randomly addressed to the SPG-OMT group and 6 to the SPG-LT group among the 13 swimmers with shoulder pain. The characteristics of the population are presented in table 1. There were no statistical significant differences between any group regarding age, height, weight, training load or training history ( $\mathrm{P}=\mathrm{NS}$ all). All the swimmers followed precisely the same training load during the protocol.

\section{Pain severity index and somatic dysfunctions}

Pain was necessarily higher in the group complaining for shoulder pain $(p<0.0001)$ at the beginning of the study but was not significantly different between SPG-OMT and SPG-LT groups at any timepoint (before/after OMT/LT, 1 week later)

\begin{tabular}{|c|c|c|c|c|c|c|}
\hline \multicolumn{6}{|c|}{ Table 1: Characteristics of the population. $(\mathrm{P}=\mathrm{NS}$ for any variable). } \\
& Sex & Age (ys) & Height $(\mathrm{cm})$ & $\begin{array}{c}\text { Weight } \\
(\mathrm{kg})\end{array}$ & $\begin{array}{c}\text { Years of } \\
\text { practice } \\
\text { (years) }\end{array}$ & $\begin{array}{c}\text { Weekly } \\
\text { training } \\
\text { amount (hs. } \\
\left.\text { wk }^{-1}\right)\end{array}$ \\
\hline CG $(\mathrm{n}=7)$ & $\begin{array}{c}3 \text { women } \\
4 \text { men }\end{array}$ & $14.4 \pm 1.3$ & $174.9 \pm 12.1$ & $60.1 \pm 10.8$ & $9.6 \pm 1.7$ & $14.4 \pm 1.3$ \\
\hline SPG-OMT $(n=7)$ & $\begin{array}{c}3 \text { women } \\
4 \text { men }\end{array}$ & $14.9 \pm 1.1$ & $175.6 \pm 7.8$ & $59.6 \pm 8.4$ & $8.7 \pm 2.1$ & $15.9 \pm 1.1$ \\
\hline SPG-LT $(n=6)$ & $\begin{array}{c}3 \text { women } \\
3 \text { men }\end{array}$ & $14.7 \pm 1.6$ & $164.8 \pm 7.3$ & $54.8 \pm 6.3$ & $7.7 \pm 2.0$ & $14.7 \pm 1.6$ \\
\hline Total $(n=20)$ & $\begin{array}{c}9 \text { women } \\
11 \text { men }\end{array}$ & $14.7 \pm 1.3$ & $172.1 \pm 10.3$ & $58.4 \pm 8.7$ & $8.7 \pm 2.0$ & $14.7 \pm 1.3$ \\
\hline
\end{tabular}


$(\mathrm{P}=\mathrm{NS})$. However, pain significantly decreased following OMT or LT in the respective groups $(6.1 \pm 1.9$ vs. $3.9 \pm 1.8 ; \mathrm{P}=0.001$ for SPG-OMT group and $6.5 \pm 1.8$ vs. $3.5 \pm 1.4 ; \mathrm{P}=0.03$ for SPGLT group) and remained stable the following week in both groups ( $\mathrm{P}=\mathrm{NS}$ comparing after treatment and 1 week later). Somatic dysfunctions were observed in both groups (Figure 1) without significant differences in the number or localization and were independent of severity of pain. Osteo-articular as well as musculo-aponevrotic dysfunctions were observed in the whole population (Figure 1), complaining or not for shoulder pain. One week after the intervention, there was a significant reduction in the recorded somatic dysfunctions by the other examiner for the SPG-OMT group but not for the 2 other groups. Table 2 present the evolution of pain.

\section{Shoulder mobility - amplitude of movement}

Shoulder's mobility was lower on the aching shoulder in the "shoulder pain" groups when compared to the control group on flexion and abduction tests but not on extension or adduction tests. Following intervention, flexion and abduction improved both in SPG-OMT and SPG-LT groups $(+12.1 /+10.1$ and $+8.0 / 5.8$ degrees respectively; $p<0.05$ both). Following intervention, only abduction improved significantly more when compared to light touch for the SPG-OMT group (Figure 2).

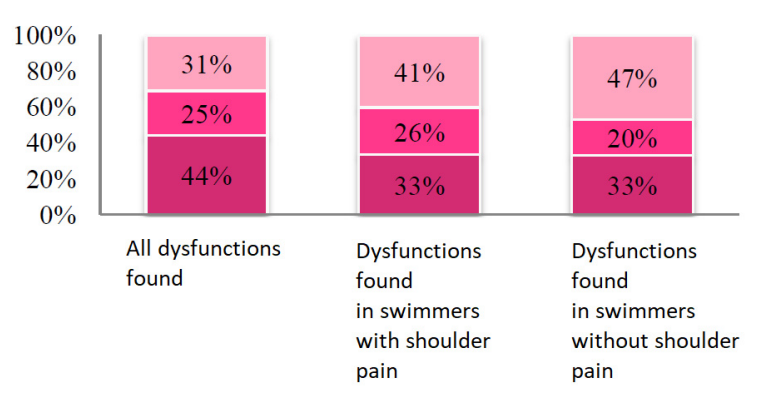

Upper extremities skeletal dysfunctions

Muscle dysfuntions

Myofascial dysunctions

Figure 1: Disfunctions found in both groups of swimmers, with and without pain.

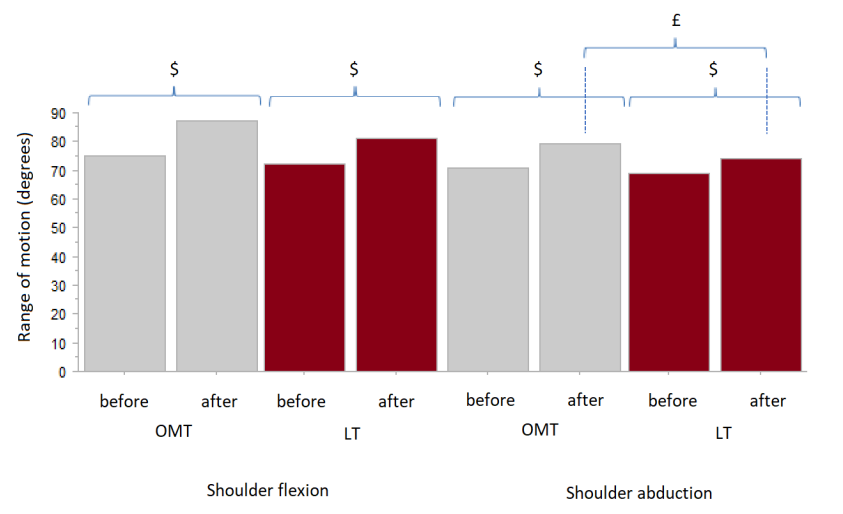

Figure 2: Flexion and abduction range of motion before and after LT/OMT. ( $\$$ stands for statistical significance before and after intervention; $£$ stands for statistical significance between OMT and light touch).
Table 2: Evolution of pain following intervention. $\mathrm{P}=\mathrm{NS}$ between groups and between "after" and "1-week later".

\begin{tabular}{|c|c|c|c|}
\hline & $\begin{array}{c}\text { Pain before } \\
\text { intervention }\end{array}$ & $\begin{array}{c}\text { Pain after } \\
\text { intervention }\end{array}$ & Pain 1-week later \\
\hline SPG-OMT & $6.1 \pm 1.9$ & $\begin{array}{c}4.1 \pm 2.2(p= \\
0.0014 \text { vs. "before") }\end{array}$ & $\begin{array}{c}3.9 \pm 1.8(p=0.0015 \\
\text { vs. "before") }\end{array}$ \\
\hline SPG-LT & $6.5 \pm 1.8$ & $\begin{array}{c}3.5 \pm 1.8(p= \\
0.0355 \text { vs. "before") }\end{array}$ & $\begin{array}{c}3.5 \pm 1.4(p=0.0308 \\
\text { vs. "before") }\end{array}$ \\
\hline
\end{tabular}

\section{Comfort of swimming}

Qualitative assessment of comfort of swimming showed a positive evolution both in SPG-OMT and SPG-LT groups and remained "unchanged" for all participants of the control group. More specifically, 6 out of 7 swimmers of the SPGOMT group reported feeling "better" and one reported feeling "unchanged" after 1 week. In the SPG-LT group, 3 swimmers reported feeling "better" and 3 reported feeling "unchanged". Nobody reported feeling "worse".

\section{Discussion}

The main result of the study is that somatic dysfunctions seem to be present, independently of the presence and/ or intensity of pain in this population of young competitive swimmers. An OMT intervention appears to reduce these somatic dysfunctions, even one week later and it looks like to be associated to evolution in comfort of swimming as shown by the qualitative reporting of comfort. Light touch yields also interesting results in the feeling of pain but with less impact on shoulder mobility and comfort of swimming. This could support the "non-specific" part of the impact of a manipulative therapy. Indeed, the swimmers felt better immediately after the intervention also because a therapist took care.

It wasn't the study's aim to address the underlying mechanisms of somatic dysfunctions or to address the origin of shoulder pain. Both student-osteopath found independently several somatic dysfunctions both at the first evaluation and one week later. The number of dysfunctions recovered was not associated to pain intensity or pain reduction in the present study. However, a decline in pain intensity along with a reduction of somatic dysfunctions following OMT was observed. Possible explanation hypotheses could be attributed to the theory of fascial manipulation during OMT [23]. Nevertheless, both OMT and LT intervention appeared positive in managing the swimmer's feeling of pain and the comfort in swimming. Pain was not associated to the number of dysfunctions recorded nor their specific localization and the non-specific effects of the intervention appear positive. Associated to the apparently positive effects in terms of range of motion of OMT, an alliance of both interventions seems positive for the swimmer.

The benefits of the intervention were still observed one week later in this specific experimental context. The swimmers did not modify anything to their training routine and were not involved in other specific physical activities. 
It would be interesting to assess the frequency and type of intervention issue in order to evaluate the preventive effects on pain appearance and injury as well as on performance. Previous retrospective studies addressed the preventive effect of OMT issue and the results were positive on stress fracture [18]. However, the preventive effects were only observed in men and it could not be concluded on the specific effects of the intervention. Further longitudinal prospective studies are needed, comparing different types of intervention and with variable frequencies.

Evaluating somatic dysfunctions is always arguable as it relies on the feeling of the operator. In this study, the two student-osteopath did not see the same participants at the first examination and one week later. They found similar somatic dysfunctions in the control group though and recorded a reduction in somatic dysfunctions in the OMT group without being aware of what the former observer recorded. They also followed the TART protocol to assess dysfunctions. Many studies have addressed inter-rater reliability in manipulative therapy and results are usually moderate like in the latest study by Consorti [24] but depending on the test, the type of tissue, joint, muscular chain (...) observed. This is consistent with other interrater reliability studies carried out in different body regions, contributing to show an overall heterogeneous level of diagnostic reliability in osteopathy. In this study, somatic dysfunction was not necessarily associated to pain intensity but an evolution in the comfort of swimming was observed with a reduction of somatic dysfunctions.

The expertise of the examiners in assessing dysfunction is also regularly argued as a limitation. The student-examiners were precisely trained but standardization among examiners can only be inferred and some variability should be expected. Nonetheless, the level of variability may also reflect the variability that would occur among osteopathic physicians. Besides, the results of this study are of limited generalizability because of the relatively small sample size.

Shoulder's pain was associated to a limited range of motion at the beginning of the present study. Following intervention (OMT/LT) swimmers gained mobility and OMT yielded significant greater improvement in the abduction movement. The specific reasons explaining this improvement could not be assessed from this study. As the etiology of pain in the swimmers' shoulder is still a debate, further studies are needed including maybe imagery. Further prospective studies exploring the preventive effect of non-invasive manipulative therapies and in order do decrease pain and increase functional movement for swimmers including the effects on the nervous system and other possible physiological effects, such as fascial changes would be of great interest for the physician, coach or athlete.

\section{Conclusion}

Somatic dysfunctions may be present in competitive swimmers. Manipulative treatment may be of potential interest in releaving pain and increasing range of mobility in this population. A better knowledge of dysfunctions associated with swimmers' shoulder can assist physicians in developing adequate preventive strategies.

\section{References}

1. https://www.nhs.uk/live-well/exercise/swimming-for-fitness/ 2019

2. Bak K. Nontraumatic glenohumeral instability and coracoacromial impingement in swimmers. Scand J Med Sci Sports. 1996; 6: 132-144. PubMed: https://www.ncbi.nlm.nih.gov/pubmed/8827842

3. Harrington S, Meisel C, Tate AA. cross sectional study examining shoulder pain and disability in division I female swimmers. J Sport Rehabil. 2014; 23: 65-75.

PubMed: https://www.ncbi.nlm.nih.gov/pubmed/23945068

4. Bulter D, Funk L, Mackenzie TA, Herrington LC. Sorting swimmer's shoulders: an observational study on swimmers that presented to a shoulder surgeon. Int J Shoulder Surg. 2015; 9: 90-93.

PubMed: https://www.ncbi.nlm.nih.gov/m/pubmed/26288538/

5. Wolf $\mathrm{BR}$, Ebinger $\mathrm{AE}$, Lawler MP, Britton $\mathrm{CL}$. Injury patterns in division I collegiate swimming. Am J Sports Med. 2009; 37: 2037-2042.

PubMed: https://www.ncbi.nlm.nih.gov/pubmed/19633232

6. Wanivenhaus F, Fox AJ, Chaudhury S, Rodeo SA. Epidemiology of injuries and prevention strategies in competitive swimmers. Sports Health. 2012; 4: 246-251.

PubMed: https://www.ncbi.nlm.nih.gov/pubmed/23016094

7. McMaster WC, Troup J. A survey of interfering shoulder pain in United States competitive swimmers. Am J Sports Med. 1993; 21: 67-70. PubMed: https://www.ncbi.nlm.nih.gov/pubmed/8427371

8. Borstad JD. Resting position variables at the shoulder: evidence to supporta posture-impairment association. Phys Ther. 2006;86:549-557. PubMed: https://www.ncbi.nlm.nih.gov/pubmed/16579671

9. Launder KG, Wenig M, Selkow NM, Williams J, Post E. Forward shoulder posture in collegiate swimmers: a comparative analysis of muscleenergy techniques. J Athl Train. 2015; 50: 1133-1139.

PubMed: https://www.ncbi.nlm.nih.gov/pubmed/26509682

10. Allegrucci M, Whitney SL, Irrgang JJ. Clinical implications of secondary impingement of the shoulder in freestyle swimmers. J Orthop Sports Phys Ther. 1994; 20: 307-318.

PubMed: https://www.ncbi.nlm.nih.gov/pubmed/7849751

11. Hanson JH, Ostrem JD, Davies BL. Effect of Kinesiology Taping on Upper Torso Mobility and shoulder Pain and Disability in US Masters National Championship Swimmers: An Exploratory Study. J Manipulative Physiol Ther. 2019; 42: 247-253.

PubMed: https://www.ncbi.nlm.nih.gov/pubmed/31221494

12. Heinlein SA, Cosgarea AJ. Biomechanical Considerations in the Competitive Swimmer's Shoulder. Sports Health. 2010; 2: 519-525. PubMed: https://www.ncbi.nlm.nih.gov/pmc/articles/PMC3438875/

13. Sein ML, Walton J, Linklater J, Harris C, Dugal T, et al. Reliability of MRI assessment of supraspinatus tendinopathy. BrJSports Med. 2007; 41:9. PubMed: https://www.ncbi.nlm.nih.gov/pubmed/17289860

14. Sein ML, Walton J, Linklater J, Appleyard R, Kirkbride, B, et al. Shoulder pain in elite swimmers: primarily due to swim-volume-induced supraspinatus tendinopathy. Br J Sports Med. 2010; 44: 105-113.

15. Struyf F, Tate A, Kuppens K, Feijen S, Michener LA. Musculoskeletal dysfunctions associated with swimmers' shoulder. Br J Sports Med. 2017; 51: 775-780.

PubMed: https://www.ncbi.nlm.nih.gov/pubmed/28189997 
16. McClure $P$, Greenberg $E$, Kareha $S$. Evaluation and management of scapular dysfunction. Sports Med Arthrosc. 2012; 20: 39-48. PubMed: https://www.ncbi.nlm.nih.gov/pubmed/22311292

17. Lewis JS. Subacromial impingement syndrome. A musculoskeletal condition or a clinical illusion? Phys Ther Rev. 2011; 11: 388-398.

18. Brumm LF, Janiski C, Balawender JL, Feinstein A. Preventive osteopathic manipulative treatment and stress fracture incidence among collegiate cross-country athletes. J Am Osteopath Assoc. 2013; 113: 882-890. PubMed: https://www.ncbi.nlm.nih.gov/pubmed/24285030

19. Ross G, Macfarlane C, Vaughan B. Combined osteopathy and exercise management of Achilles tendinopathy in an athlete. J Sports Med Phys Fitness. 2018; 58: 106-112.

PubMed: https://www.ncbi.nlm.nih.gov/pubmed/28409510

20. Brolinson PG, Smolka M, Rogers M, Sukpraprut S, Goforth MW, et al. Precompetitionmanipulativetreatmentandperformanceamong Virginia
Tech athletes during 2 consecutive football seasons: a preliminary, retrospective report. J Am Osteopath Assoc. 2012; 112: 607-615. PubMed: https://www.ncbi.nlm.nih.gov/pubmed/22984234

21. Ward RC, executive ed. Foundations for Osteopathic Medicine. 2nd ed. Philadelphia, PA: Lippincott, Williams \& Wilkins. 2002.

22. Paulet $\mathrm{T}$, Fryer $\mathrm{G}$. Inter-examiner reliability in palpation for tissue texture abnormality in the thoracic paraspinal region. IJOM. 2009; 12: 92-96.

23. Day JA, Stecco C, Stecco A. Application of fascial manipulation technique in chronic shoulder pain-anatomical basis and clinical implications. J Bodyw Mov Ther. 2009; 13: 128-135. PubMed: https://www.ncbi.nlm.nih.gov/pubmed/19329049

24. Consorti G, Basile F, Pugliese L, Petracca M. Interrater Reliability of Osteopathic Sacral Palpatory Diagnostic Tests Among Osteopathy Students. J Am Osteopath Assoc. 2018; 118: 637-644.

PubMed: https://www.ncbi.nlm.nih.gov/pubmed/30128497 